\title{
Highlights of the 27th International Papillomavirus Conference and Clinical Workshop: part 2: applied clinical science
}

Marc Arbyn*, Achim Schneider', Lutz Gissmann² \& Andreas M Kaufmann'

'Clinic for Gynecology, Charite-Universitätsmedizin Berlin, Campus Benjamin Franklin \& Campus Mitte, Berlin, Germany

2Deutsches Krebsforschungszentrum, Heidelberg, Germany

*Author for correspondence: Unit of Cancer Epidemiology, Scientific Institute of Public Health J Wytsmanstreet 14, B1050 Brussels, Belgium m Tel.: +32 26425021 = Fax: +32 26425410 m marc.arbyn@wiv-isp.be

\section{7th International Papillomavirus Conference and Clinical Workshop Berlin, Germany, 17-22 September 2011}

The 27th International Papillomavirus Conference and Clinical Workshop, held in Berlin (17-22 September 2011), brought together more than 2000 scientists, clinicians and public health experts who shared new findings in the knowledge of the HPV and the prevention and treatment of HPV-related disease. In this second of three reports of the conference, the applied clinical science sessions are summarized, which focused on immunology, new HPV tests, benign HPV infections, noncervical HPV-related disease, primary and secondary prevention of cervical cancer by HPV-based screening and prophylactic HPV vaccination and treatment of HPV-induced disease. The clinical workshops discussed possible alternative schedules of prophylactic HPV vaccination, prevention of anal cancer and anal precancer, validation of HPV genotyping assays, establishment of standards and laboratory proficiency in testing for HPV DNA and anti-HPV antibodies through the WHO LabNet, and currently heavily debated questions on the role of colposcopy in the assessment of cervical cancer precursors.

Every 12-16 months, the International Papillomavirus (IPV) Society invites the community of scientists, clinicians, public health experts and stakeholders to share new findings and experiences in the field of basic science of the papillomavirus and the prevention and treatment of HPV-related disease. At the previous conference (IPV26), organized in Montreal (3-8 July 2010) [101], the initiative was launched to summarize the highlights in a comprehensive paper published in a peerreviewed scientific journal [1]. In 2011, the 27th International Papillomavirus Conference and Clinical Workshop was held in Berlin (17-22 September) [102] and this time the sessions were condensed in a series of three papers published in Future Virology. The first report was published in the last issue of the 2011 volume of Future Virology and included the keynote lectures and the summaries of the basic science sessions [2]. The report below covers the second part of the conference devoted to applied clinical science as well as the clinical Satellite Symposia organized by stakeholders and researchers with the support of the International Papillomavirus Society. The next issue of Future Virology will contain the report on the third and last part dealing with epidemiology and public health.

\section{Applied clinical science sessions Clinical immunology (M Stanley)}

Clinical Immunology and Applied Sciences included presentations that covered central issues in the immune response to HPV. SW Lin discussed the risk of subsequent detection of HPV DNA in women seropositive but DNAnegative for that type, and the sensitivity of the available sero-assays. Protection offered by seropositivity against subsequent infection in men was not demonstrated (B Lu). Despite chronic, florid and recurrent HPV6- or HPV11-caused laryngeal warts, it was shown that $80 \%$ of children with recurrent respiratory papillomatosis (RRP) were seronegative, but seroconverted when immunized with the quadrivalent HPV vaccines (F Buchinsky). The interaction between HIV and HPV infections are topics of intense contemporary interest. T Sasagawa showed that in an area of high prevalence of HIV infection, intermediate-risk HPV types as well as high-risk types were the most frequently associated with abnormal cytology. In an elegant study from
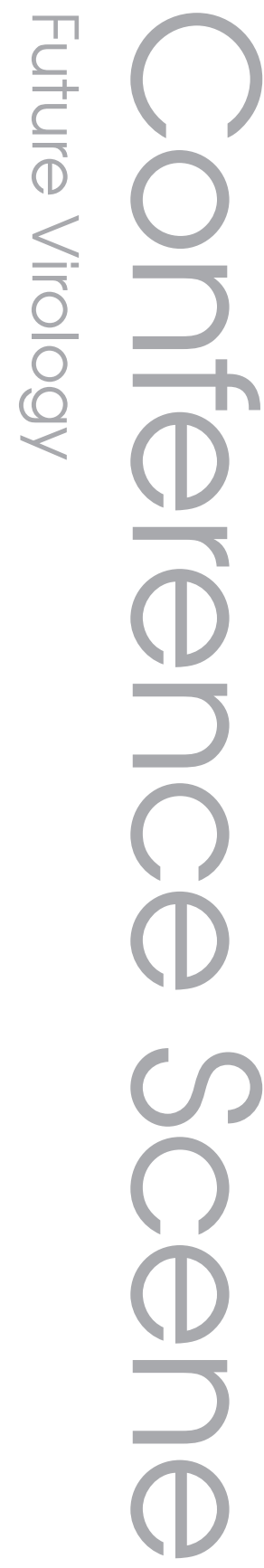

\section{Keywords}

- anal cancer = cervical cancer $=$ colposcopy $m$ head and neck cancer $m$ HPV - human papillomavirus - prophylactic vaccination - screening = treatment of AIN - treatment of CIN

\section{Future} Medicine part of 
South Africa on HPV transmission in HIV infected couples ( $Z$ Mbulawa), it was shown that low CD4 count increased the rate of HPV acquisition, with the highest risk in female-tomale transmission. Neoplastic progression in the cervix is known to be associated with increasing local immune nonresponsiveness/immunosuppression. K Torres-Poveda provided evidence that specific polymorphisms in the IL-10 promoter were associated with cervical intraepithelial neoplasia (CIN)3 and cervical cancer risk in Mexican women. Immunotherapies must modify or reverse this immunosuppression and $M$ Welters identified HPV specific Foxp $3^{+}$regulatory $\mathrm{T}$ cells as potential immune correlates of success or failure in therapeutic immunization. T Ramquist described disease specific outcomes in HPV-positive tonsillar cancer and associations with $\mathrm{CD} 8^{+} \mathrm{T}$ cell and Foxp $3^{+}$reinfiltrates.

\section{Clinical aspects of HPV testing (J Palefsky)}

K Cuschieri compared HPV E6/E7 RNA (Aptima $^{\circledR}$; Gen-Probe, CA, USA) testing with hybrid capture 2 (HC2) in women attending colposcopy clinics and showed that, while the two tests showed similar sensitivity for detection of CIN2+, the specificity of Aptima testing was slightly higher. $S$ Sorbye examined the performance of a different HPV RNA test, PreTect HPV-Proofer, for primary screening in women aged 20-69 years and showed that it could be useful to identify women at high risk of incident CIN2+. D Mesher compared different triage strategies for women with abnormal cytology (Predictor 1 and 2 studies), showing that, in general, there was a trade-off between sensitivity and specificity or positive predictive value. A Szarewski (Predictor 3 study) showed that many of the tests performed similarly with respect to sensitivity, with Aptima testing performing slightly better with respect to specificity. Similar results were presented by $\mathrm{T}$ Iftner, who compared Aptima to $\mathrm{HC} 2$ in women being screened in primary care settings in Germany. M Arbyn concluded from a meta-analysis that HC2 and Aptima show similar performance in triage of atypical squamous cells of unknown significance cytology. However, in low grade squamous intraepithelial lesion triage, Aptima was as sensitive as and more specific than $\mathrm{HC} 2$ in the detection of CIN2+. A Bailey showed similar sensitivity but better specificity of a PCR-based microarray detection system (Papillocheck $^{\circledR}$; Greiner Bio One, Frickenhausen, Germany) for subsequent detection for CIN2+ compared with $\mathrm{HC} 2$ among women with normal cytology. J van der Marel confirmed that CIN lesions isolated by laser capture microdissection from cervical biopsies almost always are due to a single HPV type, even with the presence of multiple types in the cervical smear. HPV16 was more etiologically dominant than previously thought, based on various genotype attribution models.

\section{HPV-related benign diseases (R Kirnbauer)}

J Rautava investigated the controversial issue of HPV infection in association with oral lichen planus, a chronic disorder that may develop into squamous cell cancer, especially with immunosuppressive therapy. Topoisomerase-II, caspase-3 and low-risk HPV DNA, but not high-risk HPV (hrHPV) DNA, were associated with increased risk for cancer development.

Although developed as a prophylactic vaccine, a quadrivalent HPV vaccine was applied to five juvenile and adult patients with RRP in an uncontrolled study by T Fromm. Vaccination in this therapeutic setting was immunogenic, welltolerated and followed by a reduced frequency of required surgeries as compared with the prevaccination period. It was suggested that prevention of secondary viral spread to previously uninfected sites could contribute to this effect, although it might merely reflect the natural course of disease. C Rodier identified a history of genital warts in the mother as the main epidemiologic risk factor for severity of RRP disease. An epidemiologic study from Colombian men and women (G Hernandez-Suarez) identified HPV6 and HPV11 in the majority of genital warts, and HPV16 as the most frequent high-risk type in combined HPV6 plus hrHPV infections, with no difference in genotype distribution across sex, age or number of partners. H Johansson analyzed swabs from more than 600 genital warts, using a PCR-based luminex method targeting at least 33 genital types, and additionally by rolling-circle amplification and fluorescently labeled primerPCR. By this extensive effort, a large spectrum of 34 genital HPV types were detected in $94 \%$ of genital warts, with HPV6 and HPV11 infecting $71 \%$ of warts. In addition, cutaneous HPV types were detected in a small proportion of warts. $\mathrm{K}$ Fujs Komloš examined HPV6 genomic variants in five primary and nine recurrent ano-genital warts and concluded that the recurrence of warts was a consequence of viral persistence, rather than reinfection. Genomic diversity of HPV40, HPV42, HPV43 and HPV44 low-risk types was analyzed by nucleotide sequencing of the respective $L C R, L 1$ and $E 6$. From a total of 108 
isolates, nine HPV40, 30 HPV42, three HPV43 and $19 \mathrm{HPV} 44$ genomic variants were identified (P Maver). O Richel detected high-grade anal intraepithelial neoplasia (HG-AIN) in a high proportion of anal condylomata and flat leukoplakias in HIV-positive MSM from Amsterdam examined by high-resolution anoscopy. This study confirms previous findings and underscores the urgent need for systematic anal screening in this high-risk group of patients to identify anal cancer precursor lesions in a timely manner.

\section{Clinical use of biomarkers (AB Moscicki)}

$R$ Hilfrich reported on a novel approach using the absence of L1 immunostaining as a predictor of progression. Their data demonstrated that L1-positive lesions that reflected productive HPV infection had a low malignant potential, whereas L1-negative early dysplastic lesions had a much higher risk of progression. The presentation by $\mathrm{H}$ Ikenberg of a large prospective trial of 27,000 women tackled the difficult issue of HPV-positive/cytology-normal women. P16/Ki-67-positive cytology staining was able to detect 29 of the $36(81 \%)$ CIN2+ in this group with a specificity of $79 \%$ and negative predictive value of $99 \%$. One of the most exciting findings was presented by M Schiffman and L Mirabello. In two different cohort studies, they found that methylation of specific $\mathrm{CpG}$ sites in L1 was strongly associated with progression of lesions. Two presentations examined host methylation patterns. In a prospective trial, M Einstein found that the methylation of MGMT found in biopsy samples was correlated with early CIN persistence. B Hesselink attempted to differentiate women with CIN3+ lesions from CIN2 and less by identifying methylation markers from self-collected samples. Two of the presentations looked at noncervical disease. N Phanuphak showed that hrHPV DNA and E6/E7 mRNA detection had good sensitivity and negative predictive value for HG-AIN. S Stiegler's findings suggested that biomarkers can also predict anal cancer outcome.

\section{Clinical aspects of noncervical infections (A Giuliano)}

Although the role of HPV in the aetiology of anal and oropharynx cancers is clear, many questions remain related to the reduction of disease burden for both cancers, either through screening or tailored treatments to improve outcome. In the case of nonmelanoma skin cancer (NMSC), the etiologic role of HPV remains less clear. Important questions remain, such as whether cutaneous HPV types play a direct role in skin carcinogenesis, the HPV types that are high risk for NMSC, whether these HPV types persist in the skin, and the appropriate markers to examine in studies of HPV and NMSC. Two presentations focused on cutaneous HPV. M de Koning presented data from a study of organ transplant recipients demonstrating persistence of cutaneous HPV DNA. K Anderson presented results from a sero-epidemiology study conducted in the Nordic countries, implicating HPV types 5 , 31, 38 and 76 in the development of NMSC, specifically squamous cell carcinoma of the skin. This was followed by three presentations focused on anal cancer. E Siegel presented results from a study investigating whether methylation patterns of host genes would be predictive of response to treatment with different chemotherapy regimens among anal cancer patients. O Richel and V Sahasrabuddhe presented results from studies designed to inform on tailored anal screening interventions. Risk factors for AIN and HPV genotype attribution for AIN were presented. The session ended with three presentations on the diagnosis and treatment of HPV-related oropharyngeal cancers. D Holzinger examined a panel of immunohistochemical biomarkers to assess which combination would have the highest utility in clinical practice. J Straetmans presented results from a study examining the utility of HPV testing of neck metastasis as an approach to define unknown primary tumors. Finally, R Kimple presented the development of an animal model to study the mechanism underlying the increased radiation sensitivity of HPV positive head and neck cancers.

\section{Cervical screening (J Cuzick)}

This was a wide-ranging session addressing a range of important issues in cervical screening. C Meijer showed long-term results from the Dutch study indicating long-term protection after HPV testing, and $\mathrm{H}$ Katki looked at similar issues for HPV triage of equivocal cytology. C Bergeron showed results from the PALM study of dual p16/Ki67 staining. M Almonte showed the heterogeneous nature of visual inspection after application of acetic acid (VIA), contrasting with consistent findings of HPV testing in Peru. O Alenifuga confirmed the poor performance of VIA in Nigeria. JS Smith looked at issues related to self-sampling in North Carolina. A Castenon examined the protection above age 65 from earlier cytology screening in the UK and G Ogilvie showed further round-1 data from the British Columbia HPV screening trial (CLEAR). 


\section{Prophylactic vaccination: clinical studies (J Schiller)}

$S$ Dobson reported a follow-up of the Vancouver immunogenicity trial of Gardasil, finding that two doses in girls remained noninferior to three doses in young women after 36 months, thus supporting the implementation of two-dose vaccination schedules in children. P Naud reported that Cervarix remained effective in a Brazilian cohort after 9.4 years, the longest follow-up of a viruslike particle vaccine to date. In an effectiveness trial in adolescent girls and boys, D Ferris found no cases of genital disease related to Gardasiltargeted types after approximately 4 years of follow-up. The results are encouraging but the trial size, 1800, was rather small and the number of expected events in a similar unvaccinated cohort is uncertain. In an effectiveness study of HG-AIN in MSM, S Goldstone found that the men choosing Gardasil vaccination after treatment had 50\% fewer reoccurrences than men who chose not to be vaccinated. Although encouraging, enthusiasm must be tempered because it is unclear if the men who choose to be vaccinated had comparable risk of reinfection to those who did not.

\section{Standard \& experimental treatment (W Kinney)}

In a Belgian cohort of women with a history of treatment of CIN, the risk of preterm delivery was 2.92-times higher than a matched cohort without treatment (C Simoens). M Cruickshank presented a 5-year follow-up of a large cohort of women for whom a single negative cotest (cytology and HPV) at 6 months post-treatment provided reassurance of a low recurrence rate for the ensuing 5 years. A Clad reported that the Aptima HPV test is a sensitive and specific follow-up test after treatment of CIN using Hybrid Capture 2 as the comparator. Topical treatments of high-grade CIN with imiqui$\bmod (\mathrm{C}$ Grimm), of high-grade perianal intraepithelial neoplasia in HIV-positive individuals with cidofovir (E Stier) and of HPV-positive cytologically normal women with Rebacin (CZhang) were relatively well-tolerated and effective. An in vitro study showed that siRNAs can repress HPV16 E6 and E7 expression (O Peralta-Zaragoza). The 2-year survival in a group of Kenyan women with invasive cervical cancer $(80 \%$ stage IIB+) treated with external beam radiotherapy was $<20 \%$.

\section{Satellite workshops \\ Alternate dosing schedules for HPV vaccination (G Ogilvie)}

Alternate and reduced dosing schedules of HPV vaccine are of tremendous interest, as they offer an opportunity to decrease the cost of the HPV vaccine series as well as increase overall coverage. Symposia participants presented both results of trials and programs that have implemented alternative schedules for HPV vaccine. A randomized trial of two doses of bivalent (B)-HPV vaccine at 0 and 6 months in 9-12-year-old girls found that antibody GMTs were noninferior to titers in young women aged 15-25 who received three doses after a follow-up of 24 months. Using newly detected HPV16/18 persistent infection as an end point, poststudy evaluation of 18-25 year old women who received fewer than three doses of B-HPV vaccine in the Costa Rica clinical trial found that vaccine efficacy of one or two doses was comparable to that of three doses in the shortterm (4 years after vaccination). For quadrivalent (Q)-HPV vaccine, an randomized controlled trial of 9-13-year-old girls who received two doses at month 0 and 6 found that GMTs were noninferior to 15-25-year-old young women who received three doses at month 36. Exploration of B and $T$ cells in two- versus three-dose recipients with Q-HPV vaccine revealed that the B-cell response was improved in younger participants (regardless of dose), while the T-cell response was related to number of doses. PATH reported on three alternate dosing regimens at months 0,3 and 9; 0, 6 and 12; and 0, 12 and 24 of Q-HPV vaccine in girls aged 11-13 compared with the regular dose schedule. With the exception of 0, 12 and 24, the alternate schedules achieved noninferiority for all four subtypes 1 month after the third dose. Mexico, Quebec and British Columbia have all moved to alternate dosing schedules in their programs, with all dosing at 0 and 6 months, with a third dose planned at 60 months, if judged necessary. The long term immunogenicity and efficacy of alternate dose schedules of HPV vaccine remains to be determined.

\section{New frontiers in anal cancer prevention (J Palefsky)}

$\mathrm{G}$ Clifford provided an update on increasing trends in anal cancer incidence, discussed possible explanations for the increase, and highlighted several areas and populations in which the true incidence of anal cancer was unknown. I Heard outlined recent data on anal HPV infection and AIN in women, emphasizing the relationship between cervical and anal HPV infection. A Kreuter discussed various treatment approaches to HG-AIN, highlighting favourable results with imiquimod. J Palefsky concluded that a randomized controlled trial is needed to determine the efficacy of HG-AIN treatment to reduce the 
incidence of anal cancer. He also invited all symposium attendees to join the recently established International Anal Neoplasia Society [103].

\section{HPV \& miRNA interactions (Z-M Zheng \& L Laimins)}

MicroRNAs are small regulatory RNA molecules that are transcribed from microRNA genes by cellular RNA polymerase II and regulate the expression of numerous genes at the post-transcriptional level. In the past 8 years, various efforts have been made to foster our understanding of the altered microRNA expression by oncogenic HPV infections in the development of cervical cancer and head and neck cancer. The goal of the workshop was to form a forum to update participants on the new developments in HPV and microRNA interactions in cervical tissues with HPV infections and cervical cancer. HPV31 E7 regulates the expression of miR-203 targeting p63 expression upon epithelial differentiation (L Laimins). $\mathrm{X}$ Xie reported the progressive expression of a subset of cellular miRNAs (miR-29, miR-375, miR-100) in cervical lesions and cervical cancer, and the experimental detection of microRNAs from exfoliated cervical cells. S Khan reported HPV E6 regulation of miR-218 via the histone acetyltransferase p300 and upregulation of miR363 in HPV-positive squamous cell carcinoma of the head and neck cell lines. Lastly, Z-M Zheng described the updated development of miRNA biology, regulation of cellular miRNA expression by oncogenic HPV E6 and E7, and some recent observations on cellular miRNA controls of HPV gene expression.

\section{VALGENT: clinical validation of HPV genotyping tests (M Arbyn)}

The VALGENT protocol provides a comprehensive design to validate and compare general HPV tests (identifying hrHPV infection) and HPV genotyping assays (identifying some or all high-risk types separately) using residual archived cervical cell samples from 1000 screened women enriched with 300 pathological samples (100 atypical squamous cells of unknown significance, 100 low-grade squamous intraepithelial lesions, 100 high-grade squamous intraepithelial lesions). Follow-up from the 1300 cases according to local guidelines will identify 70-120 CIN2+ cases and about 800 subjects without $\mathrm{CIN}$, allowing computation of sensitivity and specificity. One general assay and four genotyping tests were evaluated using samples derived from a Belgian biobank: $\mathrm{GP}^{+} / 6^{+} \mathrm{PCR}$ with EIA cocktail-identification of $14 \mathrm{hrHPV}$ types (M de Koning); reverse transcriptase PCR targeting viral E6/E7 genes (C Depuydt); BSGP5+/6+ PCR combined with Luminex-based multiplex HPV genotyping (M Schmidt); multiplex PCR/arrayed primer extension assay (M Tommasino); and Digene LQ Test identification of 14 separate hrHPV types (D Geraets). A second VALGENT study including six HPV assays will be set up using the Scottish HPV archive (K Cuschieri). J Dillner underlined the complementarity of clinical validation using the VALGENT protocol and the proficiency testing of HPV genotyping tests using the panel prepared by the global WHO reference laboratory.

\section{WHO HPV LabNet Satellite Workshop (J Dillner \& E Unger)}

The WHO HPV LabNet Satellite Workshop [104] was organized to review activities of global, regional and national HPV reference laboratories; review accomplishments of the WHO LabNet; and discuss methods to maintain communication and continue standardization and harmonization of HPV testing. Presentations from 11 laboratories worldwide highlighted the results of current testing, training and communication activities of laboratories involved in HPV monitoring. The accomplishments of the WHO HPV LabNet, including development of International Standards for HPV 16 and 18 DNA as well as HPV 16 antibodies, the first edition of the WHO HPV Laboratory Manual, newsletter and website, and HPV DNA proficiency testing, were made possible by a grant to the WHO from the Gates Foundation. There was enthusiastic support for continuing these standardization activities on a voluntary unfunded basis, with the WHO functioning as a facilitator of communication. The next round of HPV DNA proficiency testing was announced, with financing by user fees. Forms are available at [105]. It was agreed that plans will be made to incorporate a longer WHO HPV LabNet session at the next IPV Conference, and to discuss support with the Papillomavirus Society.

\section{Improving colposcopy in the era of HPV-based cervical cancer prevention (M Schiffman)}

W Prendiville contrasted data from the UK showing a low risk of CIN3 in the 3 years following a normal colposcopy exam with findings from the ALTS trial showing a higher risk of CIN3 in the 2 years after a negative colposcopy exam. M Gold summarized the ASCCP guidelines on colposcopy and emphasized the benefit of using static colposcopy images for training. N Wentzensen summarized findings from the Biopsy Study showing that 
increasing the number of targeted biopsies can increase detection of prevalent CIN3 by $30 \%$, but that the yield of additional random biopsies is low. $\mathrm{R}$ Pretorius summarized studies from China and the US showing that up to $40 \%$ more CIN3 can be detected using random four-quadrant biopsies. M Sideri showed a risk-based approach using colposcopy impression and cervical cancer screening results for management of women with cervical lesions. A Schneider pointed out the importance of colposcopy for planning excisional procedures. M Arbyn showed results from a meta-analysis on the performance of colposcopy-targeted punch biopsies to detect high-grade CIN assessed by excision and explained that the rather high sensitivity estimates may be inflated because of referral bias. The lively discussion focused on colposcopy training, the value of taking multiple biopsies, and the importance of prospective follow-up of colposcopically normal women.

\section{International Federation of Cervical Pathology \& Colposcopy workshop (S Tatti)}

This workshop focused on the activities and updates of the International Federation of Cervical Pathology and Colposcopy (IFCPC). The topics included were immunomodulators in the lower genital tract and therapeutic vaccines (S Tatti), the new colposcopic nomenclature approved during the IFCPC World Congress in Río de Janeiro 2011 (W Prendiville), education and standards in colposcopy worldwide (J Bentley), vulvar intraepithelial neoplasia: etiology and different treatment approaches (R Cestero and $\mathrm{H}$ Haefner), and vaccination in developing countries (P Naud).

\section{Conclusion}

In a next part of this article, to be published in an upcoming issue of Future Virology, the summaries of workshops and scientific sessions of the third part of the 27th International Papillomavirus Conference and Clinical Workshop on epidemiology and public health will be covered.

\section{Acknowledgements \\ The authors would like to thank C Schreckenberger for the logistical support in all aspects of the meeting and the correspondence with session chairs as well as the session chairs themselves who made short summaries. Finally, they would like to thank L Rudbert of Destination Oresund and their assistants for having brought together the whole community of HPV experts in the fascinating city of Berlin.}

\section{Financial \& competing interests disclosure}

MArbyn is supported by (1) the European Commission through (1a) the ECCG project (European Cooperation on development and implementation of Cancer screening and prevention Guidelines, IARC, Lyon, France), funded by DG Sanco, Luxembourg; through (1b) the PREHDICT project (grant No. 242061, coordinated by the Vrije Universiteit Amsterdam, The Netherlands) and (1c) through HPV-AHEAD (coordinated by IARC), funded by the 7th Framework Programme of DG Research, Brussels; (2) the Belgian Foundation Against Cancer (Brussels, Belgium). L Gissmann is a consultant to Qiagen, GlaxoSmithKline (GSK) and Sanofi Pasteur MSD and, due to existing intellectual property, receives royalties from sales of Gardasi $^{\circledR}$ and Cervarix ${ }^{\circledR}$. A Schneider received honoraria from GSK, Sanofi Pasteur and Karl Storz for advisory and speaking activities. AM Kaufmann has received travel grants and speakers honoraria from GSK and SP-MSD. In the past 5 years he has served as advisory board member for GSK and Gen-Probe. The authors have no other relevant affliations or financial involvement with any organization or entity with a financial interest in or financial conflict with the subject matter or materials discussed in the manuscript apart from those disclosed.

No writing assistance was utilized in the production of this manuscript.

\section{References}

1. Arbyn M, Steben M. Highlights of the 26th International Papillomavirus Conference and Workshops (Montreal, 3-8 July 2010). Future Oncol. 6, 1711-1724 (2010).

2. Arbyn M, Gissmann L, Schneider A, Kaufmann AM. Highlights of the 27th International Papillomavirus Conference and Clinical Workshop: part 1. Future Virol. 6(12), 1389-1396 (2011).

\section{Websites}

101. 26th International Papillomavirus Conference and Clinical Workshop. www.hpv2010.org

102. 27th International Papillomavirus Conference and Clinical Workshop. www.hpv2011.org

103. International Anal Neoplasia Society. (Under construction). www.iansoc.org
104. WHO HPV LabNet. www.who.int/biologicals/areas/vaccines/ hpv_labnet

105. Swedish External Quality Assessment Institute. www.equalis.se 